\title{
EL USO DIDÁCTICO Y DISCIPLINAR DE LAS TECNOLO- GÍAS EN LA FORMACIÓN INICIAL DE PROFESORES
}

\author{
Iris Caramés Beltrán ${ }^{1}$ \\ Consejo de Formación en Educación (Uruguay) \\ Instituto de Profesores "Artigas"
}

Recibido 15/05/2019 Aceptado 30/05/2019

1. Profesora de Didáctica

(Uruguay). Especialista en Educación y Nuevas Tecnologías.

Diplomada en Educación y Desarrollo. Magíster en Educación, Sociedad y Política.

Correo electrónico: iriscara@gmail.com
El artículo tiene como finalidad divulgar algunos resultados de la investigación realizada en Uruguay, entre 2017 y 2018, sobre la formación inicial de los profesores en el uso didáctico y disciplinar de las tecnologías. En primer lugar, se presentan los problemas detectados en las políticas educativas para la integración significativa de las tecnologías en las aulas y se resumen las líneas teóricas que enmarcan la investigación. Se exponen, también, la metodología y las herramientas empleadas para abordar el problema con el fin de identificar los trayectos formales cursados para la inclusión de las TIC, los contenidos y actividades propuestos en ellos y las percepciones de los estudiantes sobre sus aprendizajes y prácticas con tecnologías.

$\forall$ The purpose of the article is to disseminate some of the results of the research conducted in Uruguay between 2017 and 2018 on initial training of teachers on educational and disciplinary use of technologies. Firstly, the problems identified in education policies for the inclusion of technologies in classrooms are presented, and the conceptual lines that define the research are outlined. The methodology and the tools used for addressing the problem are also presented with the aim of identifying the formal courses taken for the integration of the ICTs, the contents and the activities proposed in them, and the students' perceptions about their learning and practices regarding technologies.

\section{DOI}

https://doi.org/10.15366/didacticas2019.20.006

PALABRAS CLAVE

Tecnologías; Didáctica específica; Formación de profesores; TIC

KEYWORDS

Technologies; Specific didactics; Training of teachers; ICT 


\section{INTRODUCCIÓN}

En este apartado, en primer lugar, se explica, sucintamente, la situación de la inclusión de las tecnologías de la información y comunicación ${ }^{1}$ en la educación como política educativa $^{2}$ y los problemas detectados, especialmente, en la formación docente inicial. En segundo lugar, se define el sintagma "uso didáctico disciplinar de las tecnologías" y en tercer lugar, lo que se entiende por la "alfabetización digital", puesto que ambos son considerados fundamentales para abordar el problema de investigación - la formación inicial del profesorado en el uso didáctico de las tecnologías- y realizar el análisis de los resultados. Por último, se describe la metodología empleada en la investigación.

\section{La integración de las TIC en la educación}

Este tema ha preocupado y ocupado a América Latina y El Caribe desde los años '90 del S. XX. Estar en la agenda de las políticas públicas de la región se debe, entre otros motivos, al potencial que tienen las tecnologías como democratizadoras del conocimiento y porque son, hoy, en la segunda década del S. XXI, fundamentales para concretar el derecho a la educación. La profusa bibliografía ${ }^{3}$ sobre políticas implementadas al respecto sostiene que se necesita avanzar con el fin de angostar las brechas digitales que se siguen dando en la región, que, según Pedró (2012) y Lugo (2010), son de acceso a Internet, de usos para alcanzar el capital cultural que genera, y de expectativas para que se pueda conectar la propuesta educativa con las necesidades del estudiante.

La primera de las tres, la de acceso, se ha reducido, en Uruguay, con el Plan Conectividad Educativa de Informática Básica para el Aprendizaje en Línea (Plan Ceibal) ${ }^{4}$ implementado en 2007, y con políticas instrumentadas por la Administración Nacional de Telecomunicaciones (ANTEL). Hasta el momento, para salvar las otras dos brechas, la de usos y la de expectativas, las políticas educativas se han centrado en formar en servicio a los docentes, lo que -según Pedró (2012), Sunkel, Trucco y Espejo (2014) y Vaillant (2013)- no

\footnotetext{
1 “Tecnologías”, “Tecnología”, “TIC” y “Tecnologías de la información y comunicación” se emplean con el significado definido por Pedró (2012): conjunto de redes, dispositivos, aplicaciones y contenidos digitales que se utilizan tanto para comunicarse con otras personas como para obtener, producir o compartir información.

2 Se entiende por política educativa la definida por Pedró y Puig (1999): resultado de la actividad de una autoridad del sector educativo investida de poder público, de legitimidad gubernamental y constituida por contenido, programa político, orientación normativa, factor de coerción y competencia social.

${ }^{3}$ Estudios del Instituto Internacional de Planeamiento de la Educación (IIPE), de la Comisión Económica para América Latina y el Caribe (CEPAL), del Fondo de las Naciones Unidas para la Infancia (UNICEF), de la Organización para la Cooperación y el Desarrollo Económico (OECD), e informes, investigaciones y análisis de autores como Pedró ( 2011, 2012), Lugo (2010), Sunkel, Trucco y Espejo (2014), Sunkel y Trucco (2012), Vaillant (2013), Coll (2008), Area Moreira (2008, 2014), Area Moreira et al (2012), Carneiro, Toscano y Díaz (2008), Dussel (2014), Báez (2014), Libâneo (2014), Rombys-Estévez (2013), Frutos (2014), Puglia (2016) y Casablancas (2016) son los consultados en la investigación.
} 
ha impactado en las aulas y, por ello, tendrían que focalizarse en la formación inicial de profesores y maestros.

El reto, entonces, de las políticas educativas de integración de la tecnología está en la formación de grado de profesores y maestros para que puedan incluirlas en sus prácticas y asimilarlas, dicho de otra manera: para hacer un uso significativo de los contenidos que la tecnología provee y aprender a transformarlo en nuevo conocimiento (Pedró, 2011).

$\mathrm{Al}$ respecto, cuatro investigaciones llevadas a cabo en Uruguay ${ }^{5}$ revelan que la formación inicial en tecnologías ha sido instrumental, escasa y sin relación entre lo aprendido y la práctica de aula. Sus análisis demuestran que las TIC aparecen desvinculadas de la didáctica y emergen como elementos independientes de los procesos de enseñanza y de aprendizaje. Por otra parte, cabe consignar que son pocos los estudios nacionales y regionales consultados que profundizan en la perspectiva de que la integración de las tecnologías en las prácticas de aula depende de la disciplina y su didáctica. Los que la proponen, algunos de manera más exhaustiva que otros, son los trabajos de Frutos (2014), de Dussel (2014), de Libâneo (2014) y de Casablancas (2016). Estos autores sostienen que la integración curricular de las TIC en la formación inicial debería plantearse a través de las materias pedagógico-didácticas que cursan los futuros docentes, con el fin de potenciar las propuestas de enseñanza. De esta manera, se imbricarían con los procesos de enseñanza para fomentar las capacidades intelectuales de los alumnos, fin primero y último de la institución educativa.

En función de lo expuesto, este artículo aborda esa perspectiva puesto que se nutre de "La formación en el uso didáctico de las tecnologías. Percepciones de estudiantes del Instituto de Profesores Artigas" 6 , investigación sobre los trayectos formativos con TIC que cursaron los estudiantes durante los cuatro años de profesorado, los contenidos enseñados en ellos y su relación con los contenidos disciplinares a enseñar, a la vez, en sus prácticas. También se pregunta cómo, cuándo y para qué utilizan las tecnologías. Estas preguntas traen aparejada la caracterización de lo que se entiende por uso didáctico disciplinar de las tecnologías.

\footnotetext{
${ }^{4}$ Fue creado en 2007 como un proyecto socioeducativo. Su objetivo es eliminar la brecha digital entre las diferentes clases sociales. Entre otras acciones, otorga computadoras portátiles a estudiantes y docentes.

5 "Integración de las TIC para una "buena enseñanza": opiniones, actitudes y creencias de los docentes en un instituto de formación de formadores", de Diego Rombys-Estévez (2013); "La inclusión del uso didáctico de las TIC en Formación Docente Inicial para Enseñanza Media. Oportunidades y desafíos en la construcción del oficio de enseñar", de Laura Frutos (2014); "La formación de estudiantes de magisterio en tecnologías digitales para la educación. La perspectiva del estudiante", de Enzo Puglia (2016) y "El uso didáctico de la tecnología en la formación de magisterio", de Silvina Casablancas (2016).

${ }^{6}$ Investigación realizada para obtener el grado de Maestría en Educación, Sociedad y Política, de la Facultad Latinoamericana de Ciencias Sociales, Programa Uruguay. Promoción: 2016 - 2018
} 


\section{El uso didáctico disciplinar de las TIC}

Definir este sintagma implica retomar la caracterización que de "Didáctica" hacen de Camilloni (2007) y Basabe (2007). Para estas autoras, es una disciplina teórica que estudia las prácticas de enseñanza, las describe, las explica y las fundamenta, lo que posibilita la enunciación de normas para intentar resolver los problemas que dichas prácticas plantean. Tiene como finalidad mejorar la enseñanza y requiere el dominio de conocimientos profesionales específicos y el despliegue de una gama de actividades y recursos que promueven procesos de aprendizaje orientados al desarrollo personal y social del estudiante. Se ocupa de una práctica, y si bien tiene una relación inmediata con los fenómenos con los que trabaja, sus productos tienen una efectividad mediada y mediata, ya que los principios didácticos deben reconstruirse en cada caso particular por actores singulares. Por ello, los docentes no pueden acomodar propuestas ajenas a sus contextos, sino que deben seleccionar, descartar opciones, decidir combinaciones. Esto supone un docente reflexivo sobre sus propias prácticas y que sabe que una misma propuesta de enseñanza a un grupo, sobre un contenido específico, no se puede extrapolar a otro, así sean del mismo grado e institución. Basabe (2007) y de Camilloni (2007) señalan, también, que la apropiación de los conocimientos y estrategias de la formación inicial en didáctica debería habilitar a los estudiantes de formación docente a:

- diseñar, implementar y evaluar programas y situaciones didácticas,

- generar estrategias de enseñanza y ambientes de aprendizaje,

- elaborar materiales didácticos para mejorar los resultados de los aprendizajes,

- ser concientes de los diversos contextos en los que se imparte la enseñanza de la asignatura,

- reflexionar sobre sus prácticas, sobre los materiales que elaboran y sobre las estrategias que emplean en sus clases para enseñar contenidos y procedimientos.

En consecuencia, se puede inferir que utilizar didácticamente las tecnologías significa emplearlas para mejorar los procesos de enseñanza y de aprendizaje de contenidos conceptuales y procedimentales. Contenidos - según cada asignatura- que deben recorrer, como propone Maggio (2012), conceptos/hechos/datos a partir de la comprensión de los marcos epistemológicos y metodológicos que dan lugar a la construcción de las teorías que sostienen y dan sentido a una disciplina ${ }^{7}$. Esto presupone -por parte del futuro docenteaprender a diseñar propuestas didácticas de sus asignaturas específicas que se entramen con las tecnologías para generar potencia pedagógica. Al respecto, sostiene Coll (2008), que son los contextos de uso y la finalidad que se persigue con la incorporación de las TIC los que determinan su capacidad para transformar la enseñanza y mejorar el aprendizaje.

\footnotetext{
${ }^{7}$ Materia, asignatura, disciplina se emplean en este documento como sinónimos.
} 
Hacer un uso didáctico disciplinar de las tecnologías requiere que el profesor (en este caso, el estudiante de profesorado) realice y, a la vez, proponga a sus propios alumnos de práctica, actividades donde estas funcionen como instrumentos psicológicos vygotskianos utilizados para planificar y regular la actividad y los procesos psicológicos, introduciendo modificaciones importantes en los procesos intra e inter-psicológicos (Coll, 2008). Un uso didáctico disciplinar implica, también, saber crear entornos que integren los sistemas semióticos conocidos y amplíen la capacidad humana para representar, procesar, transmitir y compartir los contenidos a enseñar con menos limitaciones de espacio y de tiempo, y de forma casi instantánea (Coll, 2008).

En esta misma línea de pensamiento, Litwin (2005) asevera que las prácticas de enseñanza deberían ser analizadas, reconocidas o reconstruidas a partir de las actividades de enseñanza y de aprendizaje con TIC que promuevan la reflexión en el aula. Se deduce, entonces, que la integración de las tecnologías debe hacerse desde la disciplina y que su uso didáctico depende de la relación -imbricación- con la producción de conocimiento en las prácticas de enseñanza y de aprendizaje. Asimismo, la relación entre tecnología y conocimiento debería concebirse -según Lion (2006)- como una relación de soporte-contenido para que se dé la apertura a otros modos simbólicos que enriquecen y permiten la apropiación, reelaboración y reconstrucción del conocimiento.

Por lo tanto, como la disciplina tiene una gramática que exige un abordaje didáctico específico donde las TIC deben estar imbricadas, es pertinente que el estudiante de profesorado reflexione críticamente durante toda su formación inicial sobre las estrategias para hacer un uso significativo de las tecnologías, y para ello, es fundamental que las conozca, que les sean facilitadas por sus docentes y a la vez, pueda identificarlas en sus propias prácticas.

El uso didáctico disciplinar de las tecnologías depende, entonces, de la finalidad para las que son empleadas y de las estrategias seleccionadas para generar procesos de enseñanza y de aprendizaje de la asignatura. A la vez, los materiales digitales utilizados por los docentes responsables de la formación del profesorado deberían habilitar diferentes tipos de lecturas, y por lo tanto, diferentes formas de apropiación de los contenidos disciplinares a enseñar. Un tipo de lectura a proponer es aquella en que el estudiante se transforma en coautor; otra, en la que se manifiesta en comentarios o propuestas en la interacción de la clase o en el entorno en línea, y también, una apropiación tradicional a partir de un material didáctico hipermedial que favorezca el acceso al contenido en diversos modos semióticos y con múltiples conexiones, como sostienen Schwartzman y Odetti (2011). De esta manera, se le está proporcionando, por inmersión y también de manera reflexiva, las herramientas para la apropiación significativa de los contenidos y procedimientos. Con este tipo de actividades, podrían vivenciar el uso didáctico de las tecnologías para emplearlas en sus clases prácticas, puesto que, como señalan los estudios hechos por Davini (2002), los futuros docentes, además de los conocimientos disciplinares, aprenden el modo de transmisión y de interacción de sus profesores, lo que hace deducir que, si estos no emplean 
las tecnologías en sus clases, los estudiantes no logran interiorizarlas en sus propias prácticas.

\section{La alfabetización digital}

Otra perspectiva para promover el uso significativo de las TIC en la educación es la que hace énfasis en pensarlas como tecnologías intelectuales, como estrategias de conocimiento y no solo como instrumentos de ilustración o difusión (Barbero, 1992). El empoderamiento de las TIC se logra alfabetizando con ellas. Por eso, es prioritario concebir a la alfabetización, sostienen Infante y Letelier (2013), como un derecho de las personas y un deber de las sociedades, puesto que no hay posibilidad de alcanzar una democracia efectiva mientras gran parte de la población se mantenga fuera del acceso a la lengua escrita en formato papel y en textos multimodales. En consecuencia, la formación inicial de los profesores (y también la formación continua) requiere que se entienda a la alfabetización como objeto de conocimiento y a la vez, como instrumento de apropiación de los contenidos disciplinares, lo que determina una formación en donde se reflexione sobre ella de manera específica y también transversal. No es suficiente que el futuro profesor aprenda a considerar sobre cómo lo tecnológico colabora o perjudica sus propuestas educativas y a observar cómo mejora o no el aprendizaje de sus estudiantes, sino también en concientizar -y concientizarse- sobre la importancia de la alfabetización. Es pertinente destacar que cuando se alude a generación de conocimientos se hace pensando en los disciplinares y a la enseñanza de la lectura y escritura como sus formas de aprehensión. Por lo expuesto, la alfabetización sigue siendo la finalidad de la institución educativa, ahora desde un concepto más amplio, más complejo y que requiere de otros conocimientos por parte de todos los profesores, no solo de aquellos que tienen como finalidad enseñar a leer y escribir. Ser alfabetizado hoy, como sostiene Torres (2006), es ingresar a la cultura escrita y poder y saber permanecer en ella, leyendo y escribiendo en diversos soportes en la formación inicial y continua.

Es de orden destacar que el concepto de alfabetización que se aborda en este documento se fundamenta en lo que planteó Barbero en 1992 y en lo que sostiene Dussel en 2017: leer no es aprender programación y robótica, sino entender y apropiarse de lo que se produce cultural y socialmente con el código digital. Por ello, usar la metáfora de la alfabetización para hablar de otras formas de comunicación y simbolización es riesgoso puesto que no todo lo visual ni lo sonoro es traducible a códigos lingüísticos. Son códigos distintos que pueden, a veces complementarse, y que deben enseñarse (Dussel, 2017). Al respecto, son Kress y van Leeuwen (2001) quienes dan luz a la teoría multimodal del discurso que sustenta esta concepción de la alfabetización. Consideran que en los textos que circulan por Internet se emplean varios modos semióticos combinados en su diseño para reforzarse mutuamente o cumplir roles complementarios. La comunicación es entendida, por esta teoría, como un proceso en el que un "producto" (artefacto, texto hipermedia, etc.) o evento semiótico se articula o produce y, al mismo tiempo, se interpreta y usa. Estos conceptos 
inciden en la definición del término "alfabetización" o "alfabetismo": capacidad de manejar y usar efectivamente el código de la escritura, la práctica social de leer y escribir en y con las nuevas tecnologías, saber "procesar" la información que se encuentra en los "artefactos/“textos multimodales", establecer relaciones e inferir ideas a partir de ella. Ser alfabetizado es, entonces, saber pensar a partir de un texto con diversos formatos y modos.

Por lo expuesto, entonces, y a pesar de los esfuerzos realizados por los decisores de políticas públicas, el tema sigue preocupando en la región y en Uruguay, ya que las políticas educativas implementadas hasta ahora no han logrado una inclusión significativa de las TIC en el entorno educativo. Su integración desde la didáctica disciplinar y como nuevas prácticas sociales de lectura y de escritura en la formación inicial y continua de los docentes abren nuevos caminos para que impacten en las aulas. Desde estas dos perspectivas, en forma directa e indirecta, se aborda el tema de la investigación que es insumo del presente artículo: la formación inicial del profesorado en el uso didáctico y disciplinar de las TIC.

\section{Metodología y herramientas}

Se utilizó la metodología cualitativa y se emplearon -como técnicas de recolección de datos- cuestionarios, entrevistas semiestructuradas y análisis documental. Este último consistió en el análisis de los programas de cursos obligatorios y extracurriculares de profesorado y de producciones de los estudiantes.

Es de orden señalar que, para llevar adelante la investigación, se consideró esencial partir de las representaciones sociales del conocimiento de los propios estudiantes. En primer lugar, porque ellos son los protagonistas del proceso de formación, están comprometidos con él, y en segundo lugar, porque se estima que sus percepciones conforman un tipo de conocimiento práctico que permite analizar las distancias entre el diseño de los programas de los cursos obligatorios y extracurriculares y su implementación, a partir de lo que el estudiante de profesorado piensa que se le ha enseñado y que ha aprendido. Se parte de la concepción de que la subjetividad es producida -como sostienen Rolnik y Guattari (2006)- por agenciamientos de enunciación. Indagar a partir de las percepciones es una fuente legitimada de información en la medida en que se considera a las representaciones sociales de los sujetos como una modalidad de conocimiento simbólico-emocional, generada socialmente (Alasino, 2011).

Las fuentes para recabar la información fueron las treinta y cuatro $(34)^{8}$ respuestas obtenidas de un cuestionario en línea dirigido a los estudiantes del Instituto de Profesores

\footnotetext{
${ }^{8}$ El cuestionario fue enviado a los cuatrocientos siete (407) estudiantes de Matemática, Física, Química, Ciencias Geográficas, Historia, Sociología y Filosofía que cursaban cuarto año en 2017, en el IPA. Respondieron treinta y cuatro (34), el 8,5\% del total, dato que generó nuevas interrogantes para ser desarrolladas en otra investigación.
} 
“Artigas” (IPA)9 de Matemática, Física, Química, Ciencias Geográficas, Historia, Sociología y Filosofía que cursaban cuarto año, en 2017 y de los resultados de ocho $(8)^{10}$ entrevistas semiestructuradas.

El cuestionario contenía preguntas que permitían relevar qué trayectos extracurriculares y obligatorios cursaron sobre el uso de la tecnología, qué aprendieron, qué actividades realizaron en el /los cursos, la relación entre lo aprendido con la especialidad ${ }^{11}$ y su didáctica, y las opiniones sobre lo que deberían haber aprendido para emplear las TIC con un fin didáctico.

En las entrevistas semiestructuradas, además, se investigó sobre la frecuencia de uso de las tecnologías en el aula y su empleo para la preparación de clases. También, se indagó si las TIC cumplían funciones de ampliación, explicación y /o retroalimentación de contenidos a enseñar, si se generaban con ellas entornos de aprendizaje en línea, y si se elaboraban recursos didácticos.

\section{CURSOS Y APRENDIZAJES EN LA FORMACIÓN INICIAL}

Este apartado contiene información sobre los trayectos formales curriculares y extracurriculares cursados por los estudiantes de la muestra, que tienen, entre sus objetivos, la inclusión educativa de las TIC. Luego, se describen los aprendizajes que los futuros profesores consideran que lograron en su formación inicial.

\section{Cursos}

Los trayectos formativos extracurriculares que se tuvieron en cuenta en la investigación son los que habilita el Consejo de Formación en Educación (CFE) ${ }^{12}$ y que tienen, entre otros destinatarios, a los estudiantes de profesorado: "Medios y Entornos Tecnológico-Digitales para el Aprendizaje (MEnTA)”, "Formación en Tecnologías Digitales para la Educación (FTD)”, “Aprender Tod@s” y "Flor de Ceibo”. También se indagó sobre "Infor-

\footnotetext{
${ }^{9}$ Creado en 1950, fue el primer centro de formación de profesores de Uruguay. Como consta en las actas fundacionales, imparte formación técnica y pedagógica para docentes de Enseñanza Secundaria a través de la formación en la disciplina específica, la formación pedagógica y la práctica docente. En la actualidad, y desde 2008, es uno de los institutos que forman parte del Consejo de Formación en Educación (CFE).

${ }^{10}$ Se hicieron entrevistas semiestructuradas a dos estudiantes de cuarto año de Historia y a uno de cada una de las otras asignaturas.

${ }^{11}$ Se entiende por Especialidad al profesorado elegido: Historia, Literatura, Idioma Español, Química, Geografía, Matemática, Sociología, Filosofía, Física, Astronomía, Educación visual y plástica, Educación musical, etc.

12 Órgano creado por la Ley de Educación de 2008. Es el encargado de profundizar y ampliar la formación en alguna rama del conocimiento después del egreso de $6^{\circ}$ año de Educación Secundaria o de Educación Técnico Profesional. La formación en educación abarca a maestros, maestros técnicos, profesores, y educadores sociales. Avala, también, la obtención de títulos de grado y posgrado.
} 
mática” -curso anual obligatorio- y sobre los cuatro cursos de Didáctica ${ }^{13}$.

Las respuestas de los estudiantes reflejan que "Informática" es el curso que reconocen como el responsable de su formación en tecnologías. Un solo estudiante afirma haber cursado "Medios y Entornos Tecnológico-Digitales para el Aprendizaje" (MenTA). La mayoría no conoce los trayectos extracurriculares mencionados en el cuestionario, de lo que se infiere, entonces, que los cursos que deberían formarlos para la integración de las tecnologías no es una estrategia de política educativa clave y formalmente planteada, como expresa Vaillant (2013), puesto que no cubren significativamente la matrícula, y la asignatura Informática está presente solo en tercer año de la carrera. Las respuestas a "Otros cursos" informan sobre capacitaciones de pocas horas y especialmente, instrumentales.

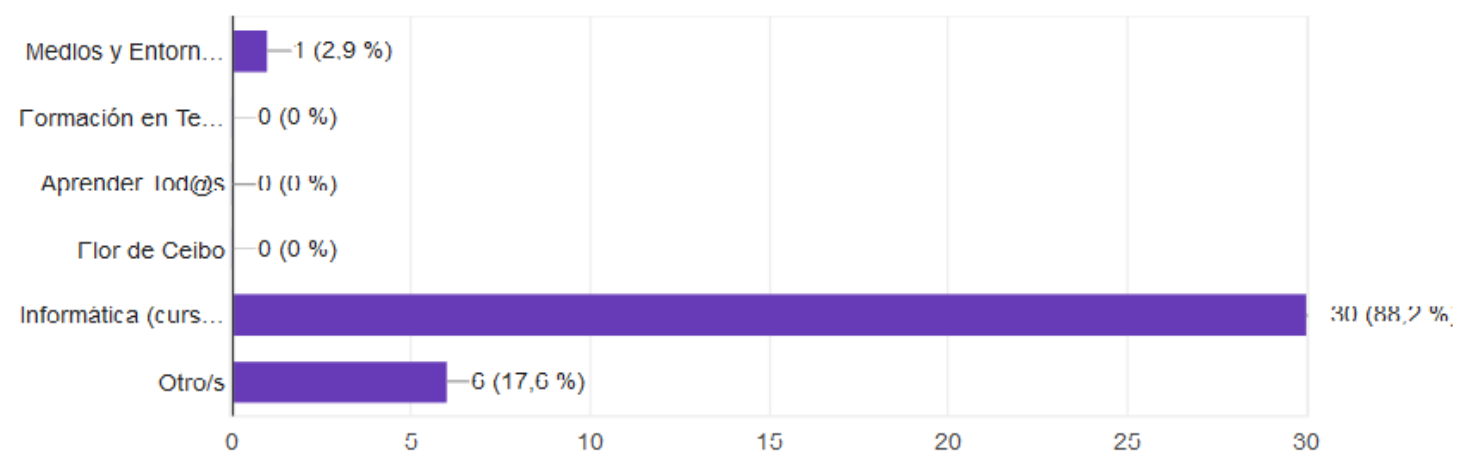

Gráfico: Número y porcentaje de estudiantes que cursaron los trayectos formales curriculares y extracurriculares. Elaboración propia. Fuente: Cuestionario en línea

Estos resultados revelan que contenidos, programas y orientación normativa para la formación en el uso didáctico de las tecnologías en la formación inicial docente no influyen significativamente. Los trayectos extracurriculares creados para paliar esta falta no impactan en su población objetivo y, por otra parte, los cuatro programas de los cursos de Didáctica de las especialidades de la muestra no desarrollan sistematizadamente el uso didáctico de las tecnologías, apenas se mencionan en una sola unidad, a excepción de Didáctica de la Matemática, que lo tiene en cuenta en varios de sus cursos.

\section{Aprendizajes}

Respecto de lo que los estudiantes consideran que han aprendido, se tomaron, como categorías de análisis, las cinco dimensiones de conocimientos que, según Area Moreira (2012), debería tener la formación del futuro profesor: a. instrumental (conocimiento práctico y

13 "Didáctica" es el nombre de la asignatura que tiene como finalidad la formación en didáctica específica de los estudiantes de profesorado. Desde 2008, se imparte a través de cuatro cursos: uno teórico, en primer año, y los otros tres, teórico-prácticos. 
estrategias de apropiación de hardware y software); b. cognitivo-intelectual (conocimientos y estrategias de búsqueda, selección, análisis, interpretación y recreación de la información); c. socio comunicacional (estrategias para la creación de textos diversos y capacidad de difundirlos, respetando las normas de convivencia del entorno tecnológico); d. axiológica (conocimiento crítico, estrategias de identificación y adquisición de valores éticos y democráticos sobre el uso de la información y de la tecnología), y e. emocional (estrategias de control emocional, empatía y construcción de la identidad digital). Para un mejor análisis, se agruparon en tres dimensiones: 1. instrumental; 2. cognitivo- intelectual y socio comunicacional y 3 . dimensión axiológica y emocional.

\section{A. DimENSIÓN INSTRUMENTAL}

Los estudiantes de la muestra sostienen que recibieron capacitación en hardware y software (sistema operativo, aplicaciones, navegación por Internet, canales de comunicación, etc.), y todos coinciden en que no fue suficiente. Demuestran confusión entre lo que les brinda el Consejo de Formación en Educación (CFE) y el Consejo de Educación Secundaria $(\mathrm{CES})^{14}$ (como la capacitación para emplear la libreta digital ${ }^{15}$ y los talleres implementados por algunas instituciones liceales), con lo que ofrece el Plan Ceibal.

Se desprende de los datos que aprenden entre ellos, de manera informal y la relación entre tecnologías y disciplina la hacen motivados por sus profesores de Informática, pero no se coordina con los profesores de Didáctica para analizar si esa relación promueve aprendizajes más significativos o si potencian la enseñanza. A propósito de ello, los estudiantes sostienen:

El programa de Informática para hacerlo efectivo debe replantearse en cuanto al uso en el aula en virtud de cada asignatura, sin dejar de lado el aprendizaje del uso en sí de la herramienta como tal, porque muchos estudiantes no lo tienen.

La dimensión instrumental, por tanto, debe potenciarse, dado que hay diferentes grados de apropiación. En el relevamiento de las preguntas al primer cuestionario, el número de estudiantes que hacía referencia a la necesidad de ser capacitados instrumentalmente ascendía a veinticinco (25) de un total de treinta y cuatro (34), lo que ratifica la necesidad de seguir capacitando al respecto, puesto que todos consideran que no han aprendido lo suficiente, al menos, sus percepciones explicitan una brecha entre lo que les es enseñado y sus

\footnotetext{
14 El Consejo de Educación Secundaria (CES) es el órgano de la Administración Nacional de Educación Pública a cargo de impartir la educación secundaria de Uruguay: media básica (Ciclo Básico) y media superior (Bachillerato).

${ }^{15}$ La libreta digital ( portafolio docente) se introdujo en el CES en 2017, y los profesores tuvieron instancias de capacitación para poder emplearla. Tiene varias prestaciones que colaboran con lo administrativo pero, sobre todo, con el seguimiento del proceso del estudiante desde que ingresa a secundaria hasta que egresa. A la vez, es un portafolio docente al que se tiene acceso desde cualquier dispositivo durante los 365 del año y que habilita compartir material, planificaciones, etc.
} 
expectativas: "Muchas veces no empleo las TIC por no saber cómo se usan los programas", sostienen, y otros hacen referencia a la necesidad de actualización constante: "Avanza rápido la tecnología, no puedo seguirla.”

De las apreciaciones de los estudiantes, se infiere la necesidad que tienen de estar informados y capacitados instrumentalmente para absorber los cambios que las tecnologías demandan, especialmente, para los usos educativos.

\section{B. DimENSIONES COGNITIVO INTELECTUAL Y SOCIO COMUNICACIONAL}

Estas dimensiones requieren el aprendizaje de estrategias para la identificación de sitios confiables, el empleo de la información, la elaboración de contenidos hipermediales, la creación de recursos didácticos digitales y el uso de plataformas educativas. Son dimensiones unidas estrechamente a lo que se ha definido como alfabetización digital.

Se constata que la mayoría de los entrevistados no elabora materiales didácticos digitales ni contenidos en diversos formatos. Sí han aprendido, algunos, a crear repositorios y a proponer actividades similares a las que se crean de manera analógica.

Cuando se analizan las producciones de los estudiantes a las que se tuvo acceso, se observa en ellas la preocupación por emplear las tecnologías y por proponer actividades en función de los contenidos conceptuales y procedimentales, pero no hay -porque no les han sido enseñados- recursos interactivos que fortalezcan los aprendizajes. Al respecto, uno de los estudiantes sostiene: "Sé que se puede trabajar interactivamente en clase, pero no lo manejo."

Asimismo, todos consideran que tendrían que aprender a crear textos en diversos formatos, lo que confirma que es, para ellos, un requisito indispensable en su formación. Algunos plantean que es imprescindible aprender a editar películas, crear videos, entornos lúdicos, etc.

Por otra parte, si bien han tenido acceso a entornos de aprendizaje generados por algunos de sus profesores, no participan, en su mayoría, de las actividades planteadas en ellos. Sostienen, en general, que se debe a que existe el temor a la exposición frente a sus pares y a los profesores. Esto determina que no vivencien, como estudiantes, las plataformas educativas y por ello, tampoco se apropien efectivamente de sus usos didácticos.

Se infiere, también, de sus respuestas, que conocen y emplean a Internet más como una biblioteca y menos como mediateca; tampoco la visualizan como gran editorial y medio de comunicación que permite el intercambio síncrono y asíncrono y que, con consignas acordes, potenciaría las estrategias de enseñanza y de aprendizaje y la alfabetización digital.

Expresan, también, que los alumnos de secundaria leen y escriben más que antes y que: "Se subestima mucho a los estudiantes, a sus mundos culturales." Por ello, afirman que se deberían dar insumos para que pudieran abordar ellos, en sus clases, a partir de esos "mun- 
dos culturales", los contenidos a enseñar. Plantean la necesidad de aprender a crear recursos desde cada asignatura y a abordar, desde la reflexión, el uso de consignas para evitar el "recorte y pegue".

Cabe destacar que en algunas especialidades (Historia, Sociología, Filosofía, Matemática) han aprendido -porque se les ha enseñado específicamente- el uso crítico de la información que provee Internet, la validación de fuentes, y por ende, se sienten seguros para enseñar esos procedimientos a sus estudiantes

\section{DiMENSIONES COGNITIVO INTELECTUAL Y SOCIO COMUNICACIONAL}

Las respuestas indican que la mayoría no ha aprendido normas de convivencia en el ecosistema de Internet, seguridad de datos ni preservación de la identidad digital. Estas dimensiones no han sido desarrolladas en los cuatro años de profesorado y por ello, muchos de los usos de las TIC en sus clases se ven restringidos (uso de redes, por ejemplo).

Acerca, entonces, de sus aprendizajes, los futuros profesores coinciden en que es menester que se les enseñe en función de su asignatura y no de manera aislada. Explicitan que las tecnologías deben asociarse con su disciplina y que se requiere orientación didáctica para hacer un uso significativo con el fin de mejorar sus estrategias de enseñanza. El juicio de uno de ellos resume lo que todos dijeron al respecto:

"La formación en TIC en mi área de especificidad es de gran ayuda, sin embargo casi todo lo aprendido resulta de la interacción [informal] con otros docentes o del aprendizaje personal."

\section{LAS TIC EN LAS PRÁCTICAS DE AULA: USOS Y DIFICULTADES}

En este apartado, se desarrollan, en primer lugar, los usos de las TIC que hacen los futuros profesores y, en segundo término, las dificultades a las que se enfrentan. Las fuentes para recabar estas informaciones han sido sus propias respuestas y el acceso a tres producciones personales, puesto que la mayoría no sistematizó la creación de recursos ni entornos en línea.

\section{Usos de las tecnologías}

El empleo de las TIC en sus clases es irregular tanto en su frecuencia como en su relevancia. Las descripciones que hacen de los usos en sus grupos de práctica demuestran esfuerzo por incorporarlas, dado que las consideran de gran valor y sobre todo, reconocen que:

"[Tenemos] que estar preparados para las nuevas generaciones de estudiantes que ya no están más adaptados al formato papel para el estudio, sino a los soportes audiovisuales.” 
A continuación, se analizan los usos en función de las categorías que describe Coll $(2008)^{16}$. Si bien este autor habla de cinco, para un mejor análisis, se agruparon en cuatro: 1. instrumentos mediadores de las relaciones entre los alumnos, los contenidos y tareas de aprendizaje; 2. instrumentos mediadores de las relaciones entre los profesores, los contenidos y las tareas de enseñanza y aprendizaje; 3 . instrumentos mediadores de las relaciones entre los profesores y los alumnos o entre los alumnos y de la actividad conjunta desplegada por profesores y alumnos y 4 . instrumentos configuradores de entornos o espacios de trabajo y de aprendizaje.

\section{A. INSTRUMENTOS MEDIADORES DE LAS RELACIONES ENTRE LOS ALUMNOS, LOS CONTENIDOS Y TA- REAS DE APRENDIZAJE}

En esta categoría, los usos más frecuentes en los estudiantes de Física y de Química es el de simuladores y, en Matemática, de Geogebra ${ }^{17}$. Expresan que los conocen porque se los mencionaron sus profesores de asignaturas específicas, o, en pocos casos, porque han tenido formación más sistematizada ${ }^{18}$ sobre su uso (especialmente Geogebra) y también, porque en Informática se les proponía que buscaran en línea para conocerlos. El uso de estos softwares cumple la función de mediar entre contenidos, alumnos y aprendizaje.

\section{B. INSTRUMENTOS MEDIADORES DE LAS RELACIONES ENTRE LOS PROFESORES, LOS CONTENIDOS Y LAS TAREAS DE ENSEÑANZA Y APRENDIZAJE}

Se observa este uso tanto en las respuestas como en las producciones a las que se tuvo acceso. Recurren a Internet para buscar contenidos, especialmente en videos o películas, y en algunos casos, en juegos para su asignatura. Los entrevistados conocen repositorios académicos para ampliar su formación en temas específicos y, a la vez, para orientar a sus estudiantes a cargo. También se apropian de algunos contenidos hipermediales para apoyar las planificaciones de clase.

\section{INSTRUMENTOS MEDIADORES DE LAS RELACIONES ENTRE LOS PROFESORES Y LOS ALUMNOS O ENTRE} LOS ALUMNOS Y DE LA ACTIVIDAD CONJUNTA DESPLEGADA POR PROFESORES Y ALUMNOS

No explicitan ningún uso que represente esta categoría.

\footnotetext{
${ }^{16}$ Para Coll (2008), las TIC tendrían que ser mediadoras de: 1 . las relaciones entre los alumnos, los contenidos y tareas de aprendizaje; 2 . los profesores, los contenidos y las tareas de enseñanza y aprendizaje; 3 . los profesores y los alumnos o entre los alumnos; 4. la actividad conjunta desplegada por profesores y alumnos durante la realización de las tareas y 5. como instrumentos configuradores de entornos o espacios de trabajo y de aprendizaje.

${ }^{17}$ Software de matemáticas para todo nivel educativo. Reúne dinámicamente geometría, álgebra, estadística y cálculo en registros gráficos, de análisis y de organización en hojas de cálculo. (Fuente: https://www.geogebra.org/)

${ }^{18}$ En "Otros cursos", algunos estudiantes plantearon que hicieron cursos cortos sobre Geogebra en ámbitos privados.
} 


\section{INSTRUMENTOS CONFIGURADORES DE ENTORNOS O ESPACIOS DE TRABAJO Y DE APRENDIZAJE}

Algunos crearon entornos en línea (páginas web y blogs). Los emplean, mayoritariamente, como repositorios y proponen actividades similares a las analógicas. Las tecnologías son usadas, en general, supliendo el medio plano (papel, pizarrón), y no se promueve interacción con los contenidos disciplinares y con el propio medio. No hay sistematización de estrategias colaborativas ni interactivas, salvo en situaciones puntuales. Nombran a CREA $^{19}$ (plataforma que provee Ceibal), Wikispaces y Edmodo. Sin embargo, no han experimentado con ellas sistematizadamente. Algunos encuentran resistencia y no logran que sus estudiantes participen (similar actitud a la que tienen algunos de ellos como estudiantes de grado). No hay explicaciones claras de por qué sucede. ¿Es un indicio de falta de hábitos? ¿La escuela primaria no fomenta el uso de las plataformas educativas? ¿Los futuros profesores no pueden usar las plataformas cuando están en el aula, que es cuando se pueden observar las dificultades o la experticia para ingresar a estos entornos educativos? Estas interrogantes fueron surgiendo en las entrevistas semiestructuradas y, por las respuestas dadas, se deduce la necesidad de reflexionar al respecto en Didáctica y de mejorar la infraestructura de las instituciones educativas en donde realizan sus prácticas.

Otro uso de las tecnologías que se corresponde con esta categoría -y que muy pocos han experimentado en sus grupos de práctica- es el de las redes para enseñar y aprender. Preguntados, mencionan a Facebook y Whatsapp. Al respecto, sostienen que Facebook les ha dado resultado en algún caso, pero solo como repositorio. Otros consideran que no usarían nunca las redes con sus estudiantes porque la distinción entre uso personal y el educativo es complejo. Solo uno de los entrevistados describe su experiencia con Whatsapp y manifiesta sorpresa del buen uso que los alumnos de primer año de Ciclo Básico ${ }^{20}$ le han dado. De sus palabras se infiere que las consignas planteadas y la interacción en esta red permitieron una sinergia enriquecedora con los contenidos, dado que muchos compartían en el grupo de Whatsapp información sobre un tema abordado en clase, incluso cuando no era ese el tema del día o de la semana. Respecto de la evaluación de esas intervenciones, era considerada como un insumo más para ver el proceso de aprendizaje y ayudaba también, a la acreditación.

\section{Dificultades para implementarlas}

Los estudiantes expresan que la dificultad que más experimentan para emplear las tecnologías en sus prácticas es de infraestructura. En algunos centros, sostienen, la infraestructura es muy buena y la conectividad también; en otros, hay problemas que van desde la

\footnotetext{
${ }^{19}$ La Plataforma "Contenidos y Recursos para la Enseñanza y el Aprendizaje” (CREA) - según Fullan (2013)es un Sistema de Gestión del Aprendizaje basado en una tecnología de "servicio integral". Ofrece recursos virtuales de enseñanza y aprendizaje como una plantilla de planificación de la clase y el acceso a los materiales de enseñanza y aprendizaje disponibles a través de los portales del Plan Ceibal.

${ }^{20}$ Ciclo Básico de Educación Secundaria (CB) se corresponde con los tres años iniciales de enseñanza media. Acceden a él los egresados de primaria.
} 
falta de enchufes en los salones, la ausencia de ordenadores portátiles para los alumnos de primer año de Ciclo Básico, pocas máquinas funcionando en las salas de informática y el escaso (a veces nulo) apoyo técnico de los profesores orientadores de informática y de tecnología (POITE) ${ }^{21}$, ya sea en la misma sala de informática como fuera de ella. Cabe aclarar que en Bachillerato $^{22}$ y en Ciclo Básico de adultos, el Plan Ceibal no entrega computadoras portátiles, lo que determina que sea más complejo emplear las tecnologías en el aula.

Asimismo, plantean que en las instituciones en las que trabajan (salvo excepciones) no hay una coordinación real entre docentes de la misma asignatura para promover el uso de las TIC. Algunos se cuestionan sus propias propuestas con tecnologías por ser conscientes de que falta reflexionar más sobre ellas desde la Didáctica, no solo como estudiantes, sino como pares en la misma institución en donde tienen sus grupos. Agregan, además, que las prácticas de evaluación instauradas en Bachillerato no permiten, durante el curso, evaluar con TIC, dado que sus estudiantes, en la instancia final del examen, se encontrarían con consignas muy diferentes para aprobarlo. Notan, también, que, a pesar de querer introducir las tecnologías, sus prácticas se ven contaminadas por las prácticas canónicas de la institución a la que pertenecen como profesores practicantes. Otra dificultad es la escasa experiencia que tienen sus propios estudiantes sobre los usos educativos de las tecnologías, lo que determina que deban explicar procedimientos instrumentales, además de los contenidos, las tareas, etc. No solo la han experimentado aquellos que han hecho sus prácticas en Bachillerato, sino también en Ciclo Básico, a pesar de que sus alumnos egresaron recientemente de primaria en donde el Plan Ceibal, instaurado en 2007, fue rápidamente absorbido por ese subsistema.

Cabe consignar que los estudiantes de secundaria, en general, no llevan (o no tienen) las computadoras móviles de Ceibal (ceibalitas) a las aulas, pero sí usan los celulares personales. La mayoría de los entrevistados que tiene grupos de primer año de Ciclo Básico afirma que les fue entregada tardíamente y que el celular las suplió desde el inicio de clases, pero no sin inconvenientes, puesto que, para tareas individuales a realizar en sus domicilios, algunos no cuentan con acceso a Internet y otros no tienen celulares inteligentes.

La siguiente transcripción de la respuesta de uno de los estudiantes que emplea la tecnología de manera sistemática revela esas dificultades que viven en el día a día en sus clases:

Desestimulan [algunos] liceos: en el liceo X tenían todo. En el liceo $\mathrm{Z}$ el escenario es diferente: no hay cañón para todos, no hay alargues, me tengo que llevar de mi casa hasta el

\footnotetext{
${ }^{21}$ El profesor orientador de informática y tecnología (POITE) tiene funciones específicas en las instituciones educativas de Secundaria, entre ellas, la de colaborar con la efectiva apropiación de las tecnologías en la institución liceal, según la Circular 29/74, del año 2010, del CES.

22 Bachillerato se corresponde con los tres años de enseñanza media superior. Acceden a él los egresados de Ciclo Básico.
} 
globo terráqueo [...]. Debo usar el cañón -cuando está libre- porque no les entregaron las ceibalitas este año. Todas en contra: todo sale a fuerza de voluntad propia.

Otro de los alumnos de profesorado resume por qué se decide emplearlas poco o, directamente, no usarlas:

Muchas veces, considerando el poco tiempo a disposición, uno prefiere no complicarse con el empleo de las TIC en los centros educativos, donde además no siempre se cuenta con todo lo necesario fácilmente accesible.

\section{OPINIONES DE LOS ESTUDIANTES SOBRE SU FORMACIÓN INICIAL}

Las percepciones sobre las dimensiones de conocimiento a aprender y sobre la relación de estos con la Didáctica específica hacen que los futuros docentes propongan que es necesario ver funcionando el uso didáctico de las tecnologías, ser ellos partícipes -como estudiantes- de ese uso, dicho de otra manera, que sus profesores de formación docente las empleen con frecuencia, y se refieren no solo a Didáctica, sino a todas las asignaturas ${ }^{23}$ :

"Son minoría aquellos docentes que usan plataformas virtuales para el trabajo en el aula." Y otros sugieren, al respecto, que: "Deberían incorporarse de forma transversal como parte de la formación integral de todo el tronco común. No solo como recursos desde la Didáctica.”

Los estudiantes de Matemática, Física y Química hacen hincapié en la necesidad del aprendizaje de softwares específicos para sus asignaturas. Los de Filosofía, Historia y Sociología consideran importante egresar habiendo aprendido a crear blogs y página web, juegos didácticos interactivos, y "textos con hipervínculos a contenidos audiovisuales y textuales” creados para sus clases. Sostienen que requieren de mayor capacitación instrumental que los de Ciencias. A la vez, los estudiantes de Ciencias necesitan estrategias de apropiación didáctica de los softwares (y de otras aplicaciones) para promover usos significativos.

De las respuestas se deduce que necesitan aprender contenidos y procedimientos según sus asignaturas y demandan que estos deben ser más profundos, y no solo los instrumentales, sino los cognitivos intelectuales y socio comunicacionales relacionados con sus actividades de enseñanza. A pesar de reconocer las diferencias en el uso de las tecnologías y

\footnotetext{
${ }^{23}$ La formación del profesorado uruguayo tiene una tradición de larga data en didáctica específica: se concreta en 1951, año en que se funda el Instituto de Profesores "Artigas" (IPA) cuya finalidad es la formación de profesores para enseñanza media de Uruguay. Desde 2008, Didáctica se imparte a través de cuatro cursos (uno teórico, en primer año, y los otros tres, teórico-prácticos). A estos cursos se suman los del Núcleo de Formación Profesional Común (NFPC): Pedagogía, Sociología de la Educación, Psicología de la Educación, Observación y Análisis de las Instituciones Educativas, Teoría del Conocimiento, Epistemología, Lengua/ Id. Español, Informática, Lenguas Extranjeras, y las materias específicas del profesorado elegido.
} 
de los diversos grados de apropiación que tienen los entrevistados, todos acuerdan en que se debe profundizar en el uso didáctico.

Los futuros profesores plantean que, a pesar de los esfuerzos de algunos docentes, no se cumplen sus expectativas: hay un distanciamiento entre la formación en didáctica disciplinar y la enseñanza del uso de las tecnologías. Los cuatro cursos de Didáctica de las especialidades de la muestra no incluyen sistematizadamente- según sus programas- el uso didáctico de las TIC, salvo Matemática. El resto tiene alguna unidad que hace referencia a ellas. A esta falta de sistematización en Didáctica, se suma la falta de uso de las TIC por parte de la mayoría de los docentes de las asignaturas específicas y de los del Núcleo de Formación Profesional Común (NFPC) ${ }^{24}$. Tampoco observan con frecuencia el uso didáctico en las clases prácticas a cargo de los profesores adscriptores: "Si el profesor adscriptor $^{25}$ no las utiliza, no tenemos el espacio para conocerlas institucionalmente”.

Sus percepciones evidencian, por lo expuesto, que la brecha de expectativas es amplia y son claros y precisos cuando exponen sus ideas y fundamentos:

[Es fundamental] integrar en las aulas de formación docente el uso de las TIC: debería estar integrado en el trabajo de cada docente para vivirlas como estudiante antes de hacerlas vivir.

Por otra parte, es de orden volver a señalar los problemas de infraestructura de diferente tipo que hay en las instituciones educativas en donde hacen sus prácticas y también, en donde asisten como estudiantes. Todos los entrevistados los mencionan y también los treinta y cuatro (34) que respondieron el cuestionario en línea. Estos escollos son los que ahondan la brecha de acceso: si no son resueltos, se seguirá postergando el uso de las tecnologías en las aulas. Si bien -en relación con otros países- se cuenta con mejor infraestructura, a nivel micro y en el día a día, los problemas existen.

Como se planteó líneas arriba, las brechas de uso y de expectativas -metáforas acuñadas por Lugo (2010) y Pedró (2012) para hacer referencia a las dificultades que se presentan para integrar las tecnologías en las aulas- deben ser angostadas en la formación inicial de profesores, tal como se ha inferido del análisis de los datos. El uso didáctico de las TIC debería depender de la formación en Didáctica. Los estudiantes así lo manifiestan cuando

\footnotetext{
${ }^{24}$ El Núcleo de Formación Profesional Común (NFPC) lo integran: Pedagogía, Sociología de la Educación, Psicología de la Educación, Observación y Análisis de las Instituciones Educativas, Teoría del Conocimiento, Epistemología, Lengua/ Id. Español, Informática, Lenguas Extranjeras.

${ }^{25}$ El docente adscriptor es un profesor de Educación Secundaria (Media) que apoya, en la práctica, al estudiante de profesorado. Son profesores adscriptores aquellos que tienen una muy buena actuación en el sistema y que, durante todo un año, tutorizan las clases prácticas del aspirante a profesor en una Institución, en un mismo curso y con el mismo grupo de alumnos. Los profesores de Didáctica I y II (responsables de los cursos teóricos) supervisan y acompañan las prácticas del aspirante a partir del trabajo conjunto con el adscriptor. En cuarto año, se cursa Didáctica III: los estudiantes tienen un grupo a cargo durante todo el año y es el profesor del curso de Didáctica III quien acompaña el proceso, no hay docente adscriptor.
} 
señalan que, para mejorar su formación, es menester tener "Clases de informática obligatorias conjuntamente con didáctica teórica y práctica." Otros plantean la necesidad de "Un aprendizaje situado en cada asignatura pues (así como en Didáctica) el uso de las TIC es muy distinto para cada asignatura, como las herramientas que pueden utilizarse."

Y también:

Una didáctica con las TIC: ¿es necesario que cada alumno esté en una computadora?, ¿en qué casos?, ¿cuándo esto sería contraproducente?, ¿cómo manejar la realidad de que, a pesar de la Ceibal, no todos tienen computadora?

Las respuestas revelan que su propia formación es un proceso al que le falta el conocimiento tecnológico de su disciplina específica para poder apropiarse de los usos didácticos de las tecnologías:

Los docentes tenemos la necesidad de incursionar en nuevos horizontes informáticos, nuestro aporte de TIC en el aula no puede ser un Power Point o un Prezi, se trata de lograr aprendizaje significativo de la mano de la tecnología y no de exponer de forma más creativa.

Estas percepciones se enmarcan en lo que Litwin, en 2005, planteó como una necesidad: la construcción de un cuerpo teórico respecto de la didáctica tecnológica. Seguir estudiando las prácticas de enseñanza dicotómicamente -tecnología/usos didácticos- no ha dado resultados puesto que la especificidad de los contenidos a enseñar /aprender parten de las epistemologías de las disciplinas. La brecha, entonces, en el uso que se les dé podría angostarse cuando se asuma que las TIC deben ensamblarse con la Didáctica disciplinar y no seguir pensándolas como algo aparte de las prácticas que suman (o no) a los procesos de enseñanza y de aprendizaje. Por ello, se considera clave que sea el campo de la Didáctica el que aborde su estudio, como disciplina teórica que estudia las prácticas de enseñanza, las describe, las explica y las fundamenta e intenta resolver los problemas que plantean, como sostiene de Camilloni (2007).

Hay otro aspecto al que los estudiantes, directa o indirectamente, hacen referencia: la alfabetización digital. Las respuestas a la pregunta "¿Qué consideras que deberías aprender en los cuatro años de tu profesorado para poder emplear las TIC con un fin didáctico?”, demuestran que deben aprender y enseñar a leer y a escribir en línea. Las siguientes transcripciones, si bien tienen un sesgo instrumental, lo verifican: "Ser capaz de crear medios audiovisuales o hacer un buen uso de distintos recursos para potenciar el trabajo en el aula." Y también: "Aprender a cortar, remixar, a seleccionar buenos recursos [ser curadores], a crear una plataforma digital para que envíen las tareas." Una de las entrevistadas resume la importancia que tiene la enseñanza del uso didáctico y disciplinar de las tecnologías dentro de un marco más amplio: 
Deberían [las tecnologías] incorporarse de forma transversal como parte de la formación integral [...]. No solo como recursos desde la Didáctica. Entre otras cosas, porque reduce el contacto al nivel forzado de la práctica en el cual intervienen otros aspectos coyunturales que no siempre condicen con las expectativas de la Didáctica. Pero si se incorporan como parte de la formación integral, se vuelve un lenguaje que puede manejarse más allá de los recursos tecnológicos disponibles en el aula.

Los diferentes usos de las tecnologías, por lo expuesto, deben ser enseñados y aprendidos para emplear efectivamente el código de la escritura y la práctica social de leer y escribir. Internet es una gran biblioteca, mediateca, medio de comunicación y editorial, por lo tanto, enseñar a leer y escribir en ella, a comunicarse y comunicar es un desafío a afrontar en la enseñanza, no solo en el nivel terciario, sino desde los inicios de la escolarización. Ser alfabetizado hoy, como sostiene Torres (2006), es ingresar a la cultura escrita y poder permanecer en ella y, también, es saber pensar a partir de un texto escrito en diversos formatos y modos (Kress y van Leeuwen, 2001). La institución educativa tiene, como fin primero y último, promover las capacidades intelectuales de los alumnos y esto se logra mediante la enseñanza continua de la lectura y de la escritura. Barbero (1992) y Dussel (2017) lo expresan claramente: leer es entender y apropiarse de lo que se produce cultural y socialmente con el código digital y no se limita a aprender programación y robótica.

El currículo de profesorado- asignaturas y seminarios numerosos- conspira contra aprendizajes más sólidos sobre el uso didáctico y disciplinar de las TIC. Por ello, los estudiantes plantean que debería revisarse para que las tecnologías estén presentes. Una de las estudiantes expresa, con preocupación, que no hay en el $\operatorname{plan}^{26}$ que se estaba elaborando al momento de la entrevista, al menos en su asignatura, ninguna referencia a las tecnologías.

Como ya se sostuvo, consideran que todos los profesores (tanto los del Núcleo Común como de las Específicas) deberían emplear las tecnologías en sus clases, para "vivirlas como estudiantes" con el fin de poder resignificarlas en sus prácticas. Agregan que, salvo excepciones, no ven en sus profesores adscriptores un uso didáctico de las tecnologías, por ello, les resulta difícil implementarlas. Para superar esta situación, sugieren un trabajo más coordinado entre profesores adscriptores y de Didáctica, en definitiva, entre profesores del CES y profesores del CFE.

Otra idea que surge es la semestralización de Informática para que se pueda abordar, en un primer semestre, lo instrumental y, en el segundo semestre, enseñar otros contenidos y procedimientos de manera coordinada con las asignaturas específicas, entre ellas, con Didáctica. Hay otros estudiantes que sugieren tener Informática los cuatro años, pero con programas acordes a las necesidades de sus profesorados y de cada Didáctica: "Creo que sería muy positivo que informática fuese una materia específica de cada especialidad.”

\footnotetext{
${ }^{26}$ Se refiere a los programas que se estaban elaborando en 2017, para formación docente.
} 
De la documentación oficial, del cuestionario en línea, de las entrevistas y de las producciones a las que se tuvo acceso, se infiere, en primer lugar, que no hay en el Plan 2008 de Formación Docente -plan vigente hoy y cursado por los estudiantes de la muestra- contenido, programa y orientación normativa que tenga entre sus cometidos la formación inicial de profesores en el uso didáctico de las tecnologías y que los cursos extracurriculares son de escaso impacto en la población objetivo de la investigación.

Los documentos analizados revelan que el curso que se relaciona más con su enseñanza es Informática, dura un año y forma parte del Núcleo de Formación Profesional Común (NFPC): todos los estudiantes de todos los profesorados cursan esta asignatura en el tercer año de su carrera y, como con cualquier otra asignatura del NFPC, pueden dar examen libre, lo que implica, para el estudiante, no participar -si así lo considera- de los procesos de aprendizaje con otros estudiantes. Informática se ocupa de la enseñanza de softwares y hardwares, pero también, aborda contenidos procedimentales en función de las especialidades, tarea nada fácil en la medida en que intereses, formación y capacitación de los estudiantes que la cursan es muy disímil. Los mismos entrevistados sustentan lo antedicho: "Se necesita más tiempo [en Informática] para investigar las aplicaciones y luego pensar la clase." Reconocen, también que "Muchos alumnos tienen un básico o nulo manejo y conocimiento de las herramientas que parecen tan básicas, para hacer [después] cualquier actividad." Otro sostiene que "No he aprendido casi nada. Lo poco que sé ha sido más el resultado de una pobre búsqueda autodidacta, o de 'piques'27 proporcionados por algún compañero.” Y también: “[La $\left.\mathrm{PAM}^{28}\right]$ es muy útil y sencilla y aprendí de rebote ${ }^{29}$ porque un compañero me comentó."

$\mathrm{Al}$ desarrollar las respuestas en la entrevista personal, sostienen que sus profesores de Informática les dan insumos, pero les resultan insuficientes porque tienen solo tres (3) horas semanales durante un año. Además, todos hacen hincapié en las dificultades que presentan ellos mismos como grupo: son heterogéneos porque hay diferentes niveles de uso de las herramientas y tienen diferentes intereses, lo que dificulta la tarea de enseñanza del profesor a cargo.

Se puede, entonces, deducir que la formación inicial de profesorado debe atender el uso de las tecnologías desde lo pedagógico, didáctico y disciplinar, propiciar una visión crítica, reflexiva y no instrumentalista de las tecnologías, y generar conciencia sobre lo que significa hoy ser alfabetizado. Por otra parte, se constata que el uso de las tecnologías separado de las prácticas educativas y de las teorías que las sustentan no ofrece aportes significativos.

\footnotetext{
27 "Piques": consejos, ideas sobre algo.

${ }^{28}$ Plataforma Adaptativa de Matemática (PAM): plataforma en línea para el aprendizaje de Matemática en Educación Primaria y Media. Se adapta al ritmo de cada estudiante y ofrece atención personalizada.

29 "De rebote": de casualidad.
} 
Si bien no todo lo que se enseña es aprendido, ni todo lo que se aprende es enseñado formalmente, las percepciones de futuros profesionales de la enseñanza, a poco de graduarse, no deja de ser revelador. Es de orden destacar la conciencia de los estudiantes de profesorado sobre la importancia del aprendizaje del uso didáctico disciplinar de las tecnologías. Esta se manifiesta tanto en las respuestas al cuestionario en línea como en las entrevistas semiestructuradas.

\section{CONCLUSIONES}

Según, entonces, los resultados de la investigación, las dificultades para la inclusión e integración de las tecnologías en la educación se corresponden con las tres brechas digitales descriptas por Lugo (2010) y Pedró (2012).

La brecha de acceso a dispositivos conectados a Internet se sigue constatando en algunas instituciones ${ }^{30}$ y especialmente, en algunos planes como los de Bachillerato y de Ciclo Básico de adultos. Para angostarla, es de orden dotar a todos los estudiantes de la Administración Nacional de Educación Pública (ANEP) ${ }^{31}$ de un dispositivo móvil y a las instituciones educativas públicas (y a cada salón de clases) brindarles una infraestructura básica, buena conectividad, y mantenimiento constante, para que se ofrezca un ámbito propicio con el fin de integrar, efectivamente, las tecnologías en las aulas.

La ausencia de una formación didáctico-tecnológica- disciplinar sistemática durante los cuatro años de la formación inicial es la que produce, entre otras causas, la brecha de usos. Se suma la ausencia de la formación en lectura y escritura digital, entendida esta como conocimiento per se y como instrumento para asir los contenidos disciplinares.

También se verifica la brecha de expectativas: hay gran distancia entre lo que requieren los futuros profesores (estar informados, capacitados y formados para absorber los cambios que las tecnologías demandan, especialmente, para los usos educativos) y lo que la formación docente les ofreció.

La presencia de estas tres brechas instan a la generación de políticas que integren el uso didáctico de las tecnologías para contener, afianzar, promover y expandir prácticas docentes, y consideren a la lectura y escritura digital como prácticas socioculturales ${ }^{32}$ fundamentales para aprender y enseñar en todos los ciclos de enseñanza, especialmente, en

\footnotetext{
${ }^{30}$ En este estudio se alude a las instituciones de enseñanza dependientes del Consejo de Educación Secundaria de Uruguay (CES) y al Instituto de Profesores "Artigas", institución dependiente del Consejo de Formación en Educación (CFE).

31 Ente autónomo con personería jurídica creado por la Ley $\mathrm{N}^{\circ} 15.739$, del 28 de marzo de 1985. Como organismo estatal, es el responsable de planificar, gestionar y administrar el sistema educativo público en el territorio uruguayo. Está conformado por todos los niveles de educación: inicial, primaria, media, técnica y terciaria.

32 Se entiende por prácticas socioculturales a las actividades humanas desarrolladas en un tiempo y un espacio dados. Esto determina que los resultados de su accionar sean específicos y únicos en función del marco
} 
la formación del profesorado. Tanto el uso didáctico de las tecnologías como la alfabetización digital demandan ser parte del cuerpo teórico de la didáctica disciplinar no solo como instrumentos, sino como parte de la epistemología de cada asignatura.

Repensar las políticas educativas de inclusión de las TIC, resignificar el concepto de alfabetización, reformular los programas de los trayectos formativos que se imparten, reflexionar sobre la necesidad de que se valore el potencial de las tecnologías en los cursos de Didáctica - puesto que su uso, separado de las prácticas educativas, hasta ahora, no ha dado resultados- son objetivos a corto plazo si se quiere influir en la integración significativa de las tecnologías en las aulas de enseñanza media.

\section{REFERENCIAS}

ALASINO, N. (2011). "Alcances del concepto de representaciones sociales para la investigación en el campo de la educación”. Revista Iberoamericana de Educación. N. ${ }^{\circ}$ 56/4.

AREA MOREIRA, M. (2008). "Educar para la sociedad informacional: Hacia el multialfabetismo". Revista portuguesa de pedagogia. Ano 42-3. 2008. pp. 7-22

—. (2014). "La alfabetización digital y la formación de la ciudadanía del siglo XXI". Revista Integra Educativa, 7 (3), pp. 21-33.

AREA MOREIRA, M., GUTIÉRREZ, A. y VIDAL, F. (2012). Alfabetización digital y competencias informacionales. Madrid: Ariel y Fundación Telefónica. Colección Fundación Telefónica, 20.

BÁEZ, M. (2014). “Uruguay”. En DUSSEL, I. (Coord). Incorporación con sentido pedagógico de TIC en la formación docente de los países del Mercosur. pp. 439 -385. Bs. As.: Teseo.

BARBERO, J. (1992). “Nuevos modos de leer”. Magazín Dominical No. 474, El Espectador. Mayo de 1992. pp. 19 - 22. Disponible en: [Enlace]. Fecha de consulta: 24/3/2019.

BASABE, L. (2007). “Acerca de los usos de la teoría didáctica”. En DE CAMILLONI, A. El saber didáctico. pp. 201-229. Buenos Aires: Paidós.

CARNEIRO, R., TOSCANO, J. y T. DÍAZ. (2008). Los desafíos de las TIC para el cambio educativo. España: OEI- Santillana. Colección Metas educativas.

CASABLANCAS, S. y GARCÍA. J. (2016). El uso didáctico de las tecnologías durante la formación de magisterio y el ejercicio docente. Facultad Latinoamericana de Ciencias

temporal y geográfico en los que se insertan. Por ejemplo, la aparición de la escritura surgió por determinadas circunstancias sociales y fue evolucionando a partir de los avances tecnológicos, sociales y culturales de las poblaciones. En consecuencia, las prácticas de lectura y de escritura varían con el tiempo y el contexto social en que se desarrollan, así como las prácticas de enseñanza. 
Sociales, sede Uruguay y Proyecto de Educación con Nuevas Tecnologías (PENT), Flacso, Argentina. Disponible en [Enlace] Fecha de consulta 20/4/2019.

COLL, C. (2008). "Aprender y enseñar con las TIC: expectativas, realidad y potencialidades”. En CARNEIRO, R, TOSCANO, J. y T. DÍAZ. Los desafíos de las TIC para el cambio educativo. pp. 113-127. España: OEI- Santillana. Colección Metas educativas.

DAVINI, M. (2002). De aprendices a maestros. Buenos Aires: Educación - Papers Editores.

DE CAMILLONI, A. (2007). El saber didáctico. Buenos Aires: Paidós.

DUSSEL, I. (Coord.) (2014). Incorporación con sentido pedagógico de TIC en la formación docente de los países del Mercosur. Bs. As.: Teseo.

-(2017). "Los analfabetos del futuro". Revista Letras Libres. Disponible en: [Enlace] Fecha de consulta: 2/1/2019.

FRUTOS, L. (2014). La inclusión del uso didáctico de las TIC en Formación Docente Inicial para Enseñanza Media. Oportunidades y desafíos en la construcción del oficio de enseñar. Tesis para obtener el título de Máster en Educación. Universidad ORT.

FULLAN, M; WATSON, N. y S. ANDERSON. (2013). Ceibal: los próximos pasos. Informe final. Toronto: Michael Fullan Enterprises.

INFANTE, M. y LETELIER, M. (2013). Alfabetización y Educación. Lecciones desde la práctica innovadora en América Latina y el Caribe. Santiago: OREALC/UNESCO.

KRESS, G. y VAN LEEUWEN, T. (2001). Multimodal discourse. The modes and media of contemporary communication. Londres: Arnold.

LIBÂNEO, J. (2014). "La integración entre el conocimiento disciplinar y el conocimiento pedagógico en la formación de profesores y la contribución de la teoría de la enseñanza de Vasili Davidov”. En Revista de Didácticas Específicas, nº 10, junio de 2014. pp. 5-37.

LION, C. (2006). Imaginar con tecnologías. Bs. As.: La Crujía.

LITWIN, E. (2005). Tecnologías educativas en tiempos de Internet. España: Amorrortu Editores.

LUGO, M. (2010). "Las políticas TIC en la educación de América Latina. Tendencias y experiencias”. Revista Fuentes, 10, 2010. pp. 52-68.

MAGGIO, M. (2012). Enriquecer la Enseñanza. Los ambientes con alta disposición tecnológica como oportunidad. Argentina: Paidós.

ORGANISATION FOR ECONOMIC CO-OPERATION AND DEVELOPMENT (OECD). (2012). Connected Minds: Technology and Today's Learners, Educational Research and Innovation. OECD Publishing. 
PEDRÓ, F. (2011). “Tecnología y escuela: lo que funciona y por qué”. En XXVI Semana Monográfica de la Educación. La Educación en la Sociedad Digital. Madrid: Fundación Santillana.

PEDRÓ, F. (2012). “¿Nuevas oportunidades, nuevas políticas para América Latina?” En SUNKEL, G.y TRUCCO, D. (2012). Las tecnologías digitales frente a los desafíos de una educación inclusiva en América Latina Algunos casos de buenas prácticas. Santiago de Chile: CEPAL, pp. 15-20.

PEDRÓ, F. Y PUIG, I. (1999). Las reformas educativas. Una perspectiva política y comparada. Barcelona: Paidós.

PUGLIA, E. (2016). La formación de estudiantes de magisterio en tecnologías digitales para la educación. La perspectiva del estudiante. Tesis para obtener el título de Máster en Educación. Universidad ORT.

ROLNIK, S. y GUATTARI, F. (2006). Micropolítica. Cartografías del deseo. Madrid: Traficantes de Sueños.

ROMBYS-ESTÉVEZ, D. (2013). "Integración de las TIC para una "buena enseñanza": opiniones, actitudes y creencias de los docentes en un instituto de formación de formadores”. Cuadernos de Investigación Educativa, Vol. 4, N. ${ }^{\circ}$ 19, 2013 pp. 69-86. Montevideo: Universidad ORT Uruguay.

SCHWARTZMAN, G. y ODETTI, V. (2011). Los materiales didácticos en la educación en línea: sentidos, perspectivas y experiencias. Ponencia presentada en ICDE-UNQ. Bs. As: PENT.FLACSO. Disponible en: [Enlace] Fecha de consulta: 13/1/2019.

SUNKEL, G. y TRUCCO, D. (2012). Las tecnologías digitales frente a los desafíos de una educación inclusiva en América Latina. Algunos casos de buenas prácticas. Santiago de Chile: CEPAL.

SUNKEL, G., TRUCCO, D. y A. ESPEJO. (2014). La integración de las tecnologías digitales en las escuelas de América Latina y el Caribe: una mirada multidimensional. Libros de la CEPAL - Desarrollo Social No. 124.

TORRES, M. (2006). “Alfabetización y aprendizaje a lo largo de toda la vida”. Revista Interamericana de Educación de Adultos, Año 28. n 1, 2006.

VAILLANT, D. (2013). Integración de TIC en los sistemas de formación docente inicial y continua para la Educación Básica en América Latina. Bs. As.: UNICEF.

\section{Documentación oficial consultada}

ADMINISTRACIÓN NACIONAL DE EDUCACIÓN PÚBLICA (ANEP). (2015). Proyecto de Presupuesto. Período 2015-2019. Disponible en [Enlace].Fecha de consulta: $23 / 4 / 2019$ 
- (2017). Marco Curricular de Referencia Nacional. Disponible en [Enlace]. Fecha de consulta: $23 / 4 / 2019$

ADMINISTRACIÓN NACIONAL DE EDUCACIÓN PÚBLICA (ANEP). CONSEJO DE FORMACIÓN EN EDUCACIÓN (CFE). (2010). Circular 29/74. Disponible en [Enlace]. Fecha de consulta: 23/4/2019

- (2008). Sistema Único Nacional de Formación Docente 2008. Disponible en [Enlace].Fecha de consulta: 23/4/2019

- (2013). Programa Medios y Entornos Tecnológico-Digitales para el Aprendizaje (MEnTA). Disponible en [Enlace].Fecha de consulta: 23/4/2019

- (2014). Programa Formación en Tecnologías Digitales para la Educación (FTD). Disponible en [Enlace].Fecha de consulta: 23/4/2019

CONSEJO DE FORMACIÓN EN EDUCACIÓN (CFE). (2008). Curso de Informática. Disponible en [Enlace] Fecha de consulta: 23/4/2019.

- (2010). Planes y Programas de Profesorado. Disponible en [Enlace]. Fecha de consulta: 23/3/2019.

- (2015). Los estudiantes de formación en educación. Estudio sobre datos aportados por el censo de estudiantes de CFE 2014-2015. Disponible en [Enlace].Fecha de consulta: 23/3/2019.

- (2017). Informe Matrícula del Consejo de Formación en Educación (CFE) - 2017. Disponible en: [Enlace]. Fecha de consulta: 23/3/2019.

MINISTERIO DE EDUCACIÓN Y CULTURA (MEC). (2009). Ley General de Educación $\mathrm{N}^{\circ}$ 18.437. Montevideo: IMPO.

PLAN CEIBAL (S/D). Programa Aprender Tod@s. Disponible en [Enlace].Fecha de consulta: $23 / 1 / 2019$

—(2017). ¿Qué es PAM? Disponible en [Enlace].Fecha de consulta: 23/1/2019

UNIVERSIDAD DE LA REPÚBLICA (S/D). Programa Flor de Ceibo. Disponible en: [Enlace]. Fecha de consulta: 23/4/2019. 